\title{
A Note on the Nullity of Unicyclic Graphs
}

\author{
Shengbiao Hu \\ Department of Mathematics, Qinghai Nationalities University, Xining, China \\ Email: shengbiaohu@aliyun.com
}

Received 17 March 2014; revised 18 April 2014; accepted 26 April 2014

Copyright (C) 2014 by author and Scientific Research Publishing Inc.

This work is licensed under the Creative Commons Attribution International License (CC BY).

http://creativecommons.org/licenses/by/4.0/

c) (i) Open Access

\section{Abstract \\ The nullity of a graph is the multiplicity of the eigenvalue zero in its spectrum. In this paper we show the expression of the nullity and nullity set of unicyclic graphs with $n$ vertices and girth $r$, and characterize the unicyclic graphs with extremal nullity.}

\section{Keywords}

Eigenvalues (of Graphs), Nullity, Unicyclic Graphs

\section{Introduction}

Let $G=(V, E)$ be a simple undirected graph with $n$ vertices. The disjoint union of two graphs $G_{1}$ and $G_{2}$ is denoted by $G_{1} \cup G_{2}$. The null graph of order $n$ is the graph with $n$ vertices and no edges. As usual, the star, path, cycle and the complete graph of order $n$ are denoted by $S_{n}, P_{n}, C_{n}$ and $K_{n}$, respectively. An isolated vertex is sometimes denoted by $K_{1}$.

Let $A(G)$ be the adjacency matrix of $G$. The eigenvalues $\lambda_{1}, \lambda_{2}, \cdots, \lambda_{n}$ of $A(G)$ are said to be the eigenvalues of $G$, and to form the spectrum of this graph. The number of zero eigenvalues in the spectrum of the graph $G$ is called its nullity and is denoted by $\eta(G)$. Let $r(G)$ be the rank of $A(G)$. Clearly, $\eta(G)=n-r(G)$.

A graph is said to be singular (nonsingular) if its adjacency matrix $A(G)$ is a singular (nonsingular) matrix.

In [1], L. Collatz and U. Sinogowitz first posed the problem of characterizing all graphs which satisfying $\eta(G)>0$. This question is of great interest in chemistry, because, as has been shown in [2], for a bipartite graph $G$ (corresponding to an alternant hydrocarbon), if $\eta(G)>0$, then it indicates the molecule which such a graph represents is unstable. The nullity of a graph is also important in mathematics, since it is related to the singularity of $A(G)$. The problem has not yet been solved completely. Some results on trees and it's line graphs, bipartite graphs, unicyclic graphs, bicyclic graphs and tricyclic graphs are known (see [3]-[14]). For details and further references we see [15] [16].

A unicyclic graph is a simple connected graph in which the number of edges equals the number of vertices. 
The length of the shortest cycle in a graph $G$ is called the girth of $G$, denoted by $g(G)$. If $G$ is a unicyclic graph, then the girth of $G$ is the length of the only cycle in $G$.

Let $U_{n}$ be the set of all unicyclic graph with $n$ vertices and let $U(n, r)$ be the set of all unicyclic graphs with $n$ vertices and girth $r$. A subset $N$ of $\{0,1,2, \ldots, n\}$ is said to be the nullity set of $U(n, r)$ provided that for any $k \in N$, there exists at least one graph $U \in V(n, r)$ such that $\eta(U)=k$, and no $k \notin N$ satisfies this property.

A matching of $G$ is a set of independent edges of $G$, a maximal matching is a matching with maximum possible number of edges. The collection of all maximal matching is denoted by $M(G)$, for any $M \in \mathcal{M}(G)$, the size of $M$, i.e., the maximum number of independent edges in $G$, is denoted by $m=m(G)$. If $n$ is even and $m=n / 2$, then we call the maximal matching a perfect matching of $G$, shot for $P M$.

It is difficult to give an expression of the nullity of a graph, so many papers give that the upper bound of the nullity of some specific graphs and characterized the extremal graphs attaining the upper bound (see [6] [9] [11] [12] [14] [17]). For the trees we know the following concise formula:

Theorem 1.1 [3] If $t$ is a tree with $n$ vertices and $m$ is the size of its maximal matchings, then its nullity is equal to $\eta(T)=n-m$.

Theorem 1.1 implies to $\eta(T)=0$ if and only if $T$ is a $P M$-tree.

In this paper we show the expression of the nullity and nullity set of unicyclic graphs with $n$ vertices and girth $r$, and characterize the unicyclic graphs with extremal nullity. For terminology and notation not defined here we refer to [3].

\section{Some Lemmas}

The following lemmas are needed, Lemmas 2.1 and Lemma 2.3 are clear.

Lemma 2.1 Let $H$ be an induced subgraph of $G$. Then $r(H) \leq r(G)$,

Lemma 2.2 Let $H$ be an induced subgraph of $G$. Then $\eta(G) \leq \eta(H)$.

Proof. $\eta(G)=n-r(G) \leq n-r(H)=\eta(H)$.

Lemma 2.3 Let $G=G_{1} \cup G_{2} \cup \cdots \cup G_{t}$, then $\eta(G)=\sum_{i=1}^{t} \eta\left(G_{i}\right)$,

where $G_{1}, G_{2}, \cdots, G_{t}$ are connected components of $G$.

Lemma $2.4[14]$

$$
r\left(C_{p}\right)= \begin{cases}n-2, & \text { if } p \equiv 0(\bmod 4) ; \\ n, & \text { if } p \neq 0(\bmod 4) .\end{cases}
$$

Let $U \in V(n, r)$, if $r=n$, then by Lemma 2.4 we have

\section{Lemma 2.5}

$$
\eta\left(C_{n}\right)=n-r\left(C_{n}\right)= \begin{cases}2, & \text { if } n \equiv 0(\bmod 4) ; \\ 0, & \text { if } n \neq 0(\bmod 4) .\end{cases}
$$

So we discuss that $r<n$ in the following unicyclics. Let $U_{0}(n, r)$ be the set of all unicyclic graphs with $n$ vertices and girth $r$ and $r<n$, let $U_{0,1}(n, r)$ be the subset of $U_{0}(n, r)$ with odd girth $r$ and let $U_{0,2}(n, r)$ be the subset of $U_{0}(n, r)$ with even girth $r$, clearly $\cup(n, r)=V_{0}(n, r) \cup\left\{C_{n}\right\}$ and $\mho_{0}(n, r)=V_{0,1}(n, r) \cup \mho_{0,2}(n, r)$.

Lemma 2.6 [3] For a graph $G$ containing a vertex of degree 1, if the induced subgraph $H$ (of $G$ ) is obtained by deleting this vertex together with the vertex adjacent to it, then the relation $\eta(H)=\eta(G)$ holds.

The characteristic polynomial of graph $G$ is denoted by

$$
\phi(G, x)=\operatorname{det}(x I-A(G))=\sum_{i=0}^{n} c_{i} x^{n-i}
$$

Lemma 2.7 [3] Let $\phi(G, x)=\sum_{i-0}^{n} c_{i} x^{n-i}$. Then the coefficient of $x^{n-i}$ is

$$
C_{i}=\sum_{H}(-1)^{k(H)} 2^{c(H)} .
$$


where the sum is over all subgraphs $H$ of $G$ consisting of disjoint edges and cycles, and having $i$ vertices. If $H$ is such a subgraph then $k(H)$ is the number of components in it and $c(H)$ is the number of cycles.

Let $i=n$ in (2), then $c_{n}=\sum_{H}(-1)^{k(H)} 2^{c(H)}$, where $H$ is spanning subgraphs of $G$ consisting of disjoint edges and cycles.

\section{Main Results}

In [18], Ashraf and Bamdad considered the opposite problem: which graphs have nullity zero? Clearly, for a graph $G, \eta(G)=0$ if and only if $c_{n} \neq 0$ and $\eta(G)>0$ if and only if $c_{n}=0$ in (1). So by (1) we have following theorem, that is

Theorem 3.1 For a graph $G$,

1) $\eta(G)=0$ if and only if $\sum_{H}(-1)^{k(H)} 2^{c(H)} \neq 0$,

2) $\eta(G)>0$ if and only if $\sum_{H}(-1)^{k(H)} 2^{c(H)}=0$.

where the sum is over all spanning subgraphs $H$ of $G$ consisting of disjoint edges and cycles.

Proof. By (1) it is clear.

By (1) we know also that $\eta(G)=n-i$ if and only if there exist $i \in\{2,3, \cdots, n\}$, such that $c_{i} \neq 0$ and $c_{i+1}=c_{i+2}=\cdots=c_{n}=0$ (Note that $c_{0}=1$ and $c_{1}=0$ ). So we have

Corollary 3.1 For a graph $G, \eta(G)=n-i=n-|V(H)|$ if and only if $\sum_{H}(-1)^{k(H)} 2^{c(H)} \neq 0$ for $|V(H)|=i$ and $\sum_{H}(-1)^{k(H)} 2^{c(H)}=0$ for $|V(H)|>i$ in (2).

Let $U$ be a unicyclic graph with girth $r$, Let $H$ be a subgraphs of $U$ consisting of disjoint edges and cycles with maximum possible number of vertices. Let $H$ be the collection of all $H$. Since $U$ is unicyclic graph, then $H$ have two types: $C_{r} \cup m\left(U-V\left(C_{r}\right)\right) P_{2}$ and $m(U) P_{2}$, where $C_{r}$ is induced subgraph of $U$ and $m P_{2}$ is disjoint union of $m$ edges $P_{2}$. Let $\mathcal{H}_{1}=\left\{C_{r} \cup m\left(U-V\left(C_{r}\right)\right) P_{2}\right\} \subset \mathcal{H}$ and $\mathcal{H}_{2}=\left\{m(U) P_{2}\right\} \subset \mathcal{H}$, clearly $\mathcal{H}_{1} \cup \mathcal{H}_{2}=\mathcal{H}$ and $\mathcal{H}_{2}=\mathcal{M}(U)$. If $r \equiv 0(\bmod 2)$, then $\left|V\left(C_{r} \cup m\left(U-V\left(C_{r}\right)\right) P_{2}\right)\right|=\left|V\left(m(U) P_{2}\right)\right|=2 m(U)$.

Since $U$ doesn't contains a subgraph $G_{1}$ consisting of disjoint edges and cycles, such that $\left|V\left(G_{1}\right)\right|>\max \left\{r+2 m\left(U-V\left(C_{r}\right)\right), 2 m(U)\right\}$, hence for $\left|V\left(G_{1}\right)\right|>\max \left\{r+2 m\left(U-V\left(C_{r}\right)\right), 2 m(U)\right\}$, $\sum_{G_{1}}(-1)^{k(H)} 2^{c(H)}=0$. So we have

Corollary 3.2 Let $U$ be a unicyclic graph with girth $r$, then $\eta(G)=n-|V(H)|$ if and only if $\sum_{H \in \mathcal{H}}(-1)^{k(H)} 2^{c(H)} \neq 0$, where $|V(H)|=\max \left\{r+2 m\left(U-V\left(C_{r}\right)\right), 2 m(U)\right\}$.

Theorem 3.2 Let $U \in V_{0}(n, r)$, then

$$
\eta(U)= \begin{cases}n-\max \left\{r+2 m\left(U-V\left(C_{r}\right)\right), 2 m(U)\right\}, & \text { if } r \equiv 1(\bmod 2) ; \\ n-2 m(U), & \text { if } r \equiv 2(\bmod 4) ; \\ n-2 m(U), & \text { if } r \equiv 0(\bmod 4) \text { and satisfies (i); } \\ n-2 m(U)+2, & \text { if } r \equiv 0(\bmod 4) \text { and satisfies (ii). }\end{cases}
$$

1) there exist $M \in \mathcal{M}(U)$, for any $r / 2$ edges in $M$, such that they not all belong to $E\left(C_{r}\right)$;

2) for any $M \in \mathcal{M}(U)$, there exist $r / 2$ edges in $M$, such that they all belong to $E\left(C_{r}\right)$.

Where $C_{r}$ is induced subgraph of $U$.

Proof. Let $U \in \mathcal{V}_{0}(n, r)$ and let $C_{r}$ be an induced subgraph of $U$. By Corollary 2.2, we only need to discuss that $\sum_{H \in \mathcal{H}}(-1)^{k(H)} 2^{c(H)}$ whether equals zero. We give a sign $e_{1}, e_{2}, \cdots, e_{r}$ for the edges of $C_{r}$, in nature order.

Case 1. $r \equiv 1(\bmod 2)$. Since $\left|C_{r} \cup m\left(U-V\left(C_{r}\right)\right) P_{2}\right|=r+2 m\left(U-V\left(C_{r}\right)\right)$ is odd and $\left|m(U) P_{2}\right|=2 m(U)$ 
is even, $\quad r+2 m\left(U-V\left(C_{r}\right)\right) \neq 2 m(U)$, hence either $H \in \mathcal{H}_{1}$ or $H \in \mathcal{H}_{2}$. If $r+2 m\left(U-V\left(C_{r}\right)\right)>2 m(U)$, then $H \in \mathcal{H}_{1}$ and $H \notin \mathcal{H}_{2}$. Since for all $H \in \mathcal{H}_{1}$, they have the same number of component, hence $\sum_{H \in \mathcal{H}}(-1)^{k(H)} 2^{c(H)} \neq 0$, where $|V(H)|=r+2 m\left(U-V\left(C_{r}\right)\right)$. If $r+2 m\left(U-V\left(C_{r}\right)\right)<2 m(U)$, then $H \in \mathcal{H}_{2}$ and $H \notin \mathcal{H}_{1}$. Similarly, $\sum_{H \in \mathcal{H}}(-1)^{k(H)} 2^{c(H)} \neq 0$, where $|V(H)|=2 m(U)$. Thus $\eta(U)=n-\max \left\{r+2 m\left(U-V\left(C_{r}\right)\right), 2 m(U)\right\}$.

Case 2. $r \equiv 2(\bmod 4)$.

Subcase 2.1 There exist $H_{0} \in \mathcal{H}_{1}$, where $H_{0}=C_{r} \cup m\left(U-V\left(C_{r}\right)\right) P_{2}$. In this case, the $H_{1}=e_{1} \cup e_{3} \cup \cdots \cup e_{r-1} \cup m\left(U-V\left(C_{r}\right)\right) P_{2}=m(U) P_{2} \in \mathcal{H}_{2} \subset \mathcal{H}$ and $H_{2}=e_{2} \cup e_{4} \cup \cdots \cup e_{r} \cup m\left(U-V\left(C_{r}\right)\right) P_{2}=m(U) P_{2} \in \mathcal{H}_{2} \subset \mathcal{H}$, where the $m\left(U-V\left(C_{r}\right)\right) P_{2}$ in $H_{0}, H_{1}$ and $H_{2}$ are same, and we call $H_{1}$ and $H_{2}$ are conjugate subgraph of $H_{0}$. Since $r / 2$ is odd, hence for any $H \in \mathrm{H}$, the number of component of $H$ have the same odevity, hence $\sum_{H \in \mathcal{H}}(-1)^{k(H)} 2^{c(H)} \neq 0$, where $|V(H)|=2 m(U)$.

Subcase 2.2 There doesn't exist $H \in \mathcal{H}_{1}$. In this case, since all $H \in \mathcal{H}_{2} \subset \mathcal{H}$ and they have the same edges,

hence $\sum_{H \in \mathcal{H}}(-1)^{k(H)} 2^{c(H)} \neq 0$, where $|V(H)|=2 m(U)$. So $\eta(U)=n-2 m(U)$.

Case 3. $r \equiv 0(\bmod 4)$ and there exist $M \in \mathcal{M}(U)$, for any $r / 2$ edges in $M$, such that they not all belong to $E\left(C_{r}\right)$.

Subcase 3.1 There exist $H_{0} \in \mathrm{H}_{1}$, where $H_{0}=C_{r} \cup m\left(U-V\left(C_{r}\right)\right) P_{2}$. In this case, the $H_{1}=e_{1} \cup e_{3} \cup \cdots \cup e_{r-1} \cup m\left(U-V\left(C_{r}\right)\right) P_{2}=m(U) P_{2} \in \mathcal{H}_{2} \subset \mathcal{H}$ and $H_{2}=e_{2} \cup e_{4} \cup \cdots \cup e_{r} \cup m\left(U-V\left(C_{r}\right)\right) P_{2}=m(U) P_{2} \in \mathcal{H}_{2} \subset \mathcal{H}$. Let $\mathcal{H}^{\prime}=\left\{H_{0}, H_{1}, H_{2}\right\} \subset \mathcal{H}$. For $H \in \mathcal{H}^{\prime}$, we have

$$
\begin{aligned}
\sum_{H \in \mathcal{H}}(-1)^{k(H)} 2^{c(H)} & =(-1)^{1+m\left(U-V\left(C_{r}\right)\right)} 2+(-1)^{r / 2+m\left(U-V\left(C_{r}\right)\right)}+(-1)^{r / 2+m\left(U-V\left(C_{r}\right)\right)} . \\
& =(-1)^{1+m\left(U-V\left(C_{r}\right)\right)} 2+(-1)^{r / 2+m\left(U-V\left(C_{r}\right)\right)} 2=0
\end{aligned} .
$$

Since we know that there exist $M \in \mathcal{M}(U)$, for any $r / 2$ edges in $M$, such that they not all belong to $E\left(C_{r}\right)$, hence we assume that $M=H_{3}\left(=m(U) P_{2}\right) \in \mathcal{H}_{2}$ and for any $r / 2$ edges in $H_{3}$, such that they not all belong to $E\left(C_{r}\right)$. Except $H_{3}$, if there exist others $H_{i} \in \mathcal{H}_{2} \quad(i \geq 4)$ and for any $r / 2$ edges in $H_{i}(i \geq 4)$, such that they not all belong to $E\left(C_{r}\right)$, then we have

$$
\begin{aligned}
\sum_{H \in \mathcal{H}}(-1)^{k(H)} 2^{c(H)}= & (-1)^{1+m\left(U-V\left(C_{r}\right)\right)} 2+(-1)^{r / 2+m\left(U-V\left(C_{r}\right)\right)}+(-1)^{r / 2+m\left(U-V\left(C_{r}\right)\right)} \\
& +(-1)^{m(U)}+(-1)^{m(U)}+\cdots \\
= & (-1)^{m(U)}+(-1)^{m(U)}+\cdots \neq 0
\end{aligned}
$$

and $|V(H)|=2 m(U)$, so $\eta(U)=n-2 m(U)$.

Subcase 3.2 There aren't exist $H \in \mathcal{H}_{1}$. In this case, similar to Subcase 2.2 of Case 2, we have $\eta(U)=n-2 m(U)$.

Case 4. $r \equiv 0(\bmod 4)$ and for any $M \in \mathcal{M}(U)$, there exist $r / 2$ edges in $M$, such that they all belong to $E\left(C_{r}\right)$. In this case, for any $M=H_{1} \in \mathcal{H}_{2}$, let $H_{1}=e_{1}^{\prime} \bigcup e_{2}^{\prime} \cup \cdots \cup e_{r / 2}^{\prime} \cup m\left(U-V\left(C_{r}\right)\right) P_{2}$, where $e_{i}^{\prime}(i=1,2, \cdots, r / 2)$ is independent edges in $C_{r}$. For the same $m\left(U-V\left(C_{r}\right)\right) P_{2}$ with $H_{1}$, let $H_{2}=e_{r / 2+1}^{\prime} \cup e_{r / 2+2}^{\prime} \cup \cdots \cup e_{r}^{\prime} \cup m\left(U-V\left(C_{r}\right)\right) P_{2}$ and $H_{0}=C_{r} \cup m\left(U-V\left(C_{r}\right)\right) P_{2}$, where $e_{r / 2+i}^{\prime}(i=1,2, \cdots, r / 2)$ is also independent edges in $C_{r}$, then $H_{2} \in \mathcal{H}_{2}$ and $H_{0} \in \mathcal{H}_{1}$. In fact, in this case for any one $H^{\prime} \in \mathcal{H}_{2}$, there exist a conjugate graph $H^{\prime \prime}\left(\in \mathcal{H}_{2}\right)$ of $H^{\prime}$, such that $H \in \mathcal{H}_{1}$, where $H^{\prime}$ and $H^{\prime \prime}$ are conjugate subgraphs of $H$, that is $V(H)=V\left(H^{\prime}\right)=V\left(H^{\prime \prime}\right)$ and $E(H)=E\left(H^{\prime}\right) \cup E\left(H^{\prime \prime}\right)$. Similarly, for any one $H \in \mathcal{H}_{1}$, it corres- 
ponding two conjugate subgraphs $H^{\prime}, H^{\prime \prime} \in \mathcal{H}_{2}$. So

$$
\sum_{H \in \mathcal{H}}(-1)^{k(H)} 2^{c(H)}=(-1)^{1+m\left(U-V\left(C_{r}\right)\right)} 2+(-1)^{r / 2+m\left(U-V\left(C_{r}\right)\right)}+(-1)^{r / 2+m\left(U-V\left(C_{r}\right)\right)}+\cdots=0,
$$

where $|V(H)|=2 m(U)$. Since $\sum_{H \in \mathcal{H}}(-1)^{k(H)} 2^{c(H)}=0$ if $|V(H)|=2 m(U)$, thus we consider the subgraph $H$ of $U$ consisting of disjoint edges and cycles, and having $m(U)-1$ edges. Clearly there exist a

$(m(U)-1)$-matching, such that there exist $r / 2-1$ edges belong in $E\left(C_{r}\right)$ and $m\left(U-V\left(C_{r}\right)\right)$ edges belong in $U-V\left(C_{r}\right)$. Similar to Case 3, $\sum_{H \in \mathcal{H}}(-1)^{k(H)} 2^{c(H)} \neq 0$, where $|V(H)|=2(m(U)-1)$. So $\eta(U)=n-2 m(U)+2$.

Let $C_{r}$ be a cycle and let $P_{n-r}$ be a path. Suppose that $v$ is a vertex of $C_{r}$ and $u$ is a pendant vertex of $P_{n-r}$. Joining $v$ and $u$ by an edge, the resulting graph (Figure 1) is denoted by $U(r, n-r)$.

$$
\text { Corollary 3.3 Let } U \in V_{0}(n, r) \text {, then } \eta(U) \leq \begin{cases}n-r-1, & \text { if } r=1(\bmod 2) ; \\ n-r, & \text { if } r=0(\bmod 2) \text {. }\end{cases}
$$

Proof. Since $r<n$, hence $U$ contains an induced subgraph $U(r, 1)$ (see Figure 1).

Case 1. $r \equiv 1(\bmod 2)$. In this case, by Theorem 2.2 we have $\eta(U(r, 1))=n-\max \left\{r+2 m\left(U(r, 1)-V\left(C_{r}\right)\right), 2 m(U(r-1))\right\}=n-2 m(U(r-1))=n-r-1$, by Lemma 2.2 we have $\eta(U) \leq n-r-1$.

Case 2. $r \equiv 0(\bmod 2)$. In this case, if $r \equiv 2(\bmod 4)$, by Theorem 2.2 we have $\eta(U(r, 1))=n-2 m(U(r, 1))=n-r$. If $r \equiv 0(\bmod 4)$, then there exist $M \in \mathcal{M}(U(r, 1))$, such that the pendant edge belong to $M$, that is for any $r / 2$ edges in $M$, it not all belong to $E\left(C_{r}\right)$, so $\eta(U(r, 1))=n-2 m(U(r, 1))=n-r$, by Lemma 2.2 we have $\eta(U) \leq n-r$.

Let $r=3$ if $r$ is odd and let $r=4$ if $r$ is even in Corollary 2.3, and combine to Lemma 2.7 we have

Corollary 3.4 [18] For any $U \in \mathcal{V}_{n}(n \geq 5), \eta(U) \leq n-4$.

Corollary 3.5 Let $U \in V_{0,1}(n, r)$, then $\eta(U)=0$ if and only if $n$ is even and $U$ contains $P M$ or $n$ is odd and $U-V\left(C_{r}\right)$ contains $P M$.

Proof. Let $U \in V_{0,1}(n, r)$, where $r$ is odd.

“ $\Rightarrow$ ' If $\eta(U)=0$, then by Theorem 2.2 we have $\max \left\{r+2 m\left(U-V\left(C_{r}\right)\right), 2 m(U)\right\}=n$.

Case 1. If $n$ is even, then $2 m(U)=n, U$ contains $P M$.

Case 2. If $n$ is odd, then $r+2 m\left(U-V\left(C_{r}\right)\right)=n, 2 m\left(U-V\left(C_{r}\right)\right)=n-r, U-V\left(C_{r}\right)$ contains $P M$. " $\Leftarrow$ "

Case 1. If $n$ is even and $U$ contains $P M$, then $\max \left\{r+2 m\left(U-V\left(C_{r}\right)\right), 2 m(U)\right\}=2 m(U)=n$, by Theorem 2.2, $\eta(U)=0$.

Case 2. If $n$ is odd and $U-V\left(C_{r}\right)$ contains $P M$, then $\max \left\{r+2 m\left(U-V\left(C_{r}\right)\right), 2 m(U)\right\}=r+2 m\left(U-V\left(C_{r}\right)\right)=r+(n-r)=n$, by Theorem 2.2, $\eta(U)=0$.

Corollary 3.6 Let $U \in V_{0,2}(n, r)$, then $\eta(U)=0$ if and only if $n \equiv 2(\bmod 4)$ and $U$ contains $P M$ or $n \equiv 0(\bmod 4)$ and $U$ contains $P M$, and for any $r / 2$ edges in the $P M$, such that they not all belong to $E\left(C_{r}\right)$.

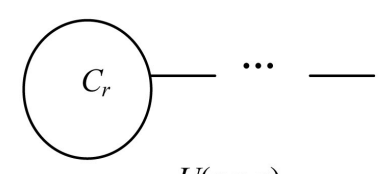

$U(r, n-r)$

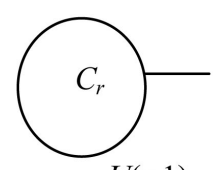

$U(r, 1)$

Figure 1. The unicyclic graph $U(r, n-r)$ and $U(r, 1)$. 
Proof. Let $U \in \mathcal{V}_{0,2}(n, r)$, where $r$ is even.

“ $\Rightarrow$ " If $\eta(U)=0$, then by theorem 2.2 we have $n-2 m(U)=0$ or $n-2 m(U)+2=0$. If $n-2 m(U)+2=0$, then $m(U)=n / 2+1$, a contradiction. So we have $n-2 m(U)=0, U$ contains $P M$. Since $r$ is even, hence $r \equiv 2(\bmod 4)$ or $r \equiv 0(\bmod 4)$. If $r \equiv 0(\bmod 4)$, then there exist $P M$, for any $r / 2$ edges in the $P M$, such that they not all belong to $E\left(C_{r}\right)$. Otherwise, by Theorem 2.2 we have $n-2 m(U)+2=0$, a contradiction.

" $\Leftarrow$ "

Case 1. If $r \equiv 2(\bmod 4)$ and $U$ contains $P M$, then by Theorem 2.2 we have $\eta(U)=n-2 m(U)=0$.

Case 2. If $r \equiv 0(\bmod 4)$ and $U$ contains $P M$, and for any $r / 2$ edges in the $P M$, such that it not all belong to $E\left(C_{r}\right)$, then by Theorem 2.2 we have $\eta(U)=n-2 m(U)=0$.

An edge belonging to a matching of a graph $G$ is said to cover its two end-vertices. A vertex $v$ is said to be perfectly covered $(P C)$ if it is covered in all maximal matching of $G$ [7].

Any vertex adjacent to a pendent vertex is a $P C$-vertex. However, there may be exist $P C$-vertices adjacent to no pendent vertex. For instance, the central vertex in the path on an odd number of vertices is $P C$.

Let $v_{i}(i=1,2, \cdots,\lceil r / 2\rceil)$ be the $P C$-vertices of $C_{r}$. Let $U_{r}^{\prime}$ be a graph is obtained from $C_{r}$, by adding $r_{i}$ $\left(0 \leq r_{i} \leq n-r\right)$ pendant edges in the $P C$-vertex $v_{i}(i=1,2, \cdots,\lceil r / 2\rceil)$ of $C_{r}$, respectively. Where $\sum_{i=1}^{\lceil r / 2\rceil} r_{i}=n-r>0$. The degree of $P C$-vertices of $U_{r}^{\prime}$ needn't equality, even for some $P C$-vertices, no pendant vertex joint to the $P C$-vertex, but the sum of number of all pendant vertices is $n-r$. For $r=5$ and 6 , an $U_{5}^{\prime}$ and $U_{6}^{\prime}$ see Figure 2, the $P C$-vertices are indicated by numbers 1, 2, 3.

Let $V_{1}^{\prime}(n, r)$ be the set of all $U_{r}^{\prime}$, where $r$ is odd and let $V_{2}^{\prime}(n, r)$ be the set of all $U_{r}^{\prime}$, where $r$ is even. Clearly $V_{1}^{\prime}(n, r) \subset V_{0,1}(n, r)$ and $V_{2}^{\prime}(n, r) \subset V_{0,2}(n, r)$. For any $U \in V_{1}^{\prime}(n, r)(i=1,2)$, the $P C$-vertices of $C_{r}$ is also the $P C$-vertices of $U$, where $C_{r}$ is inducted subgraph of $U$.

Let $d(v, G)$ denote the distance from a vertex $v$ to the graph $G$, if $v \in V(G)$, then $d(v, G)=0$.

Corollary 3.7 Let $U \in \mathcal{V}_{0,1}(n, r)$, then $\eta(U)=n-r-1$ if and only if $U \in \mathcal{V}_{1}^{\prime}(n, r)$.

Proof. Since $U \in \mathcal{V}_{0,1}(n, r)$, hence $r$ is odd.

“ $\Rightarrow$ " Let $U \in V_{0,1}(n, r)$, if $\eta(U)=n-r-1$, by Theorem 2.1 we have $\max \left\{r+2 m\left(U-V\left(C_{r}\right)\right), 2 m(U)\right\}=r+1$. Since $r$ is odd, hence $2 m(U)=r+1, m(U)=(r+1) / 2$, so for any pendant $v$ of $U, d\left(v, C_{r}\right) \leq 2$. Otherwise, $m(U) \geq(r+3) / 2$, a contradiction. If there exist at least one pendant vertex $v$ in $U$, such that $d\left(v, C_{r}\right)=2$, then there exist at least one independent edge in $U-V\left(C_{r}\right)$, so $\max \left\{r+2 m\left(U-V\left(C_{r}\right)\right), 2 m(U)\right\} \geq r+2 m\left(U-V\left(C_{r}\right)\right) \geq r+2, \quad \eta(U) \leq n-r-2<n-r-1$, a contradiction. So for any pendant vertex of $U, d\left(v, C_{r}\right)=1$. Since there exist $(r+1) / 2 P C$-vertices in $C_{r}$, if there exist pendant edges for every vertices of $C_{r}$ in $U$, then $\max \left\{r+2 m\left(U-V\left(C_{r}\right)\right), 2 m(U)\right\}=2 m(U)=2 r>r+1$, a contradiction. Hence there exist pendant edges for part of vertices of $C_{r}$ in $U$. If there exist $(r+1) / 2+1$ vertices in $C_{r}$ such that every vertex have pendant edges, then $\max \left\{r+2 m\left(U-V\left(C_{r}\right)\right), 2 m(U)\right\} \geq 2[(r+1) / 2+1]>r+1$, a contradiction. So there exist at most $(r+1) / 2$ vertices, such that every vertex have pendant edges, that is all pendant vertices of $U$ joint to at most $(r+1) / 2$ vertices in $C_{r}$. In the neighbor vertices of all pendant vertices of $U$, if there exist $(r-1) / 2 P C$-vertices and one non $P C$-vertex of $C_{r}$, then

$\max \left\{r+2 m\left(U-V\left(C_{r}\right)\right), 2 m(U)\right\} \geq 2 m(U) \geq 2(m(U(r, 1))+1)=2((r+1) / 2+1)>r+1$, a contradiction.

Thus all pendant vertices of $U$ are joint to the $P C$-vertices of $C_{r}$, thus $U \in \mathcal{V}_{1}^{\prime}(n, r)$.

“ $\Leftarrow$ " Let $U \in \mathcal{V}_{1}^{\prime}(n, r)$ (see Figure 2), since $r$ is odd, $r+2 m\left(U-V\left(C_{r}\right)\right)=r$ and $2 m(U)=r+1$, hence $\max \left\{r+2 m\left(U-V\left(C_{r}\right)\right), 2 m(U)\right\}=r+1$, by Theorem 2.1, we have 


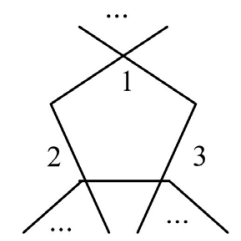

$U_{5}{ }^{\prime}$

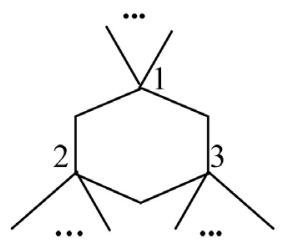

$U_{6}^{\prime}$

Figure 2. An $U_{5}^{\prime}$ and an $U_{6}^{\prime}$, its $P C$-vertices are indicated by numbers $1,2,3$.

$\eta(U)=n-\max \left\{r+2 m\left(U-V\left(C_{r}\right)\right), 2 m(U)\right\}=n-r-1$.

Let $u$ be a vertex of $C_{r}$, and let $v$ be a $k$-degree vertex of $K_{1, k+1}$. Joining $u$ and $v$ by a path $P_{1}$, the resulting graph is denoted by $U(r, l, k+1)$, where $r+l+k=n$. When $l=2$, we get $U(r, 2, k+1)$ (Figure 3 ).

For convenience, we call the star in $U(r, 2, k+1)$ is pendant star. Let $U^{\prime}(r, l, k)$ be a unicyclic graph come from $U\left(r, l, k+1\right.$ ), by removing a pendant edge and adding it to another vertex of $C_{r}$, where $r+l+k=n$ (See Figure 4).

Corollary 3.8 Let $U \in V_{0,2}(n, r)$, then $\eta(U)=n-r$ if and only if $U \in V_{2}^{\prime}(n, r)$ or $U \cong U(r, 2, k+1)$ and $r \equiv 0(\bmod 4)$

Proof. Since $U \in V_{0,2}(n, r)$, hence $r$ is even.

“ $\Rightarrow$ " Let $U \in \mathcal{V}_{0,2}(n, r)$, if $\eta(U)=n-r$, by Theorem 2.2 we have $2 m(U)=r$ or $2 m(U)-2=r$.

Case 1. $2 m(U)=r$. In this case, since $r$ is even, hence for any pendant $v$ of $U, d\left(v, C_{r}\right) \leq 1$. Otherwise, $m(U) \geq r / 2+1$, a contradiction. For an edge $u v \in E\left(C_{r}\right)$, If $u$ and $v$ both have at lest one pendant edge in $U$, respectively. Then $m(U) \geq r / 2+1$, a contradiction. So all pendant vertices of $U$ join to some $P C$-vertices of $U$, thus $U \in V_{2}^{\prime}(n, r)$.

Case 2. $2 m(U)-2=r$. In this case $m(U)=r / 2+1$, since $r$ is even, hence for any one pendant $v$ of $U$, $d\left(v, C_{r}\right) \leq 3$. Otherwise, $m(U) \geq r / 2+2$, a contradiction.

Subcase 2.1. There exist $v \in U$, such that $d\left(v, C_{r}\right)=3$. In this case, $U(n, 3)$ (see Figure 1 ) is an induced subgraph of $U$, then there exist $M \in \mathcal{M}(U(n, 3))$, such that the pendant edge belong to $M$, so for any $r / 2$ edges in $M$, it not all belong to $E\left(C_{r}\right)$, by Theorem 2.1 we have $\eta(U(n, 3))=n-2 m(U(n, 3))=n-r-2$, by Lemma 2.2, $\eta(U) \leq \eta(U(n, 3))=n-r-2$, a contradiction.

Subcase 2.2. There exist $v \in U$, such that $d\left(v, C_{r}\right)=2$. In this case, $U(r, 2, k+1)$ (see Figure 3, specially take $k=0$ ) is an induced subgraph of $U$, and only one vertex of $U$ have only one pendant star. Otherwise $m(U) \geq r / 2+2$, a contradiction. If there exist at lest one pendant edge in other one vertex of $C_{r}$, the resulting graph is denoted by $U^{\prime}(r, 2, k)$ (see Figure 4). Since there exist $M \in \mathcal{M}\left(U^{\prime}(r, 2, k)\right)$, such that the two independent pendant edges in $\left(U^{\prime}(r, 2, k)\right)$ belong to $M$, we know that $m\left(U^{\prime}(r, 2, k)\right)=r / 2+1$, hence for any $r / 2$

edges in $M$, they not all belong to $E\left(C_{r}\right)$, by Lemma 2.2 and Theorem 2.2 we have $\eta(U) \leq \eta\left(U^{\prime}(r, 2, k)\right)=n-2 m\left(U^{\prime}(r, 2, k)\right)=n-r-2$, a contradiction. So $U \cong U(r, 2, k+1)$ (see Figure 3). If $r \equiv 2(\bmod 4)$, by Theorem 2.2 we have $\eta(U(r, 2, k+1))=n-2 m(U(r, 2, k+1))=n-r-2$, a contradiction. So $U \cong U(r, 2, k+1)$ and $r \equiv 0(\bmod 4)$.

" $\Leftarrow$ " Case 1. Let $U \in \mathcal{V}_{2}^{\prime}(n, r)$ (see Figure 2), since $r$ is even, hence $2 m(U)=r$. If $r \equiv 2(\bmod 4)$, then by Theorem 2.1 we have $\eta(U)=n-2 m(U)=n-r$. If $r \equiv 0(\bmod 4)$, since $r<n$, hence $U$ contains a induced subgraph $U(r, 1)$ (see Figure 1), for a $M \in \mathcal{M}(U(r, 1))$, let the pendant edge of $U(r, 1)$ belong to the $M$, then the $r / 2$ edges in $M$, not all belong to $E\left(C_{r}\right)$, by Theorem 2.2 we have $\eta(U)=n-2 m(U)=n-r$.

Case 2. Let $U \cong U(r, 2, k+1)$ and $r \equiv 0(\bmod 4)$, then $m(U(r, 2, k+1))=r / 2+1$. Since for any 


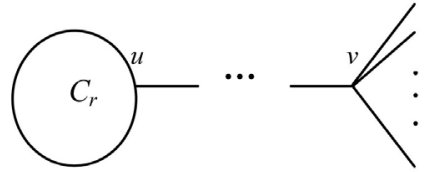

$U(r, l, k+1)$

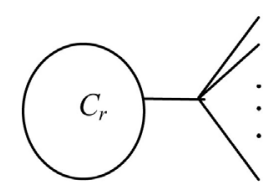

$U(r, 2, k+1)$

Figure 3. The unicyclic graphs $U(r, l, k)$ and $U(r, 2, k)$.

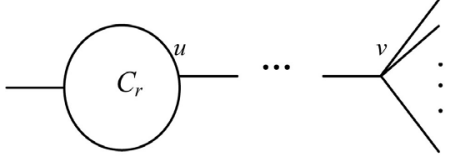

$U^{\prime}(r, l, k)$

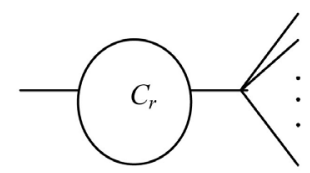

$U^{\prime}(r, 2, k)$

Figure 4. The unicyclic graphs $U^{\prime}(r, l, k)$ and $U^{\prime}(r, 2, k)$.

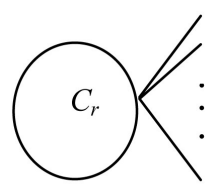

$U^{\prime}(r, 1, k+1)$

Figure 5. The unicyclic graph $U(r, 1, k+1)$.

$M \in \mathcal{M}(U(r, 2, k+1))$, there exist $r / 2$ edges in $M$, such that they all belong to $E\left(C_{r}\right)$, by Theorem 2.1 we have $\eta(U(r, 2, k+1))=n-2 m(U(r, 2, k+1))+2=n-r$.

Let $l=1$ in $U(r, l, k+1)$ (Figure 3 ), we get the following graph $U(r, 1, k+1)$ (Figure 5).

Theorem 3.3 The nullity set of $\mathrm{U}_{0,1}(n, r)$ is $\{0,1,2, \ldots, n-r-1\}$.

Proof. By Corollary 2.3, we only need to show that for each $k \in\{0,1,2, \cdots, n-r-1\}$, there exist a unicyclic graph $U \in \mathcal{V}_{0,1}(n, r)$ such that $\eta(U)=k$, where $r$ is odd.

Case 1. $k=0$. Let $U=U(r, n-r)$ (see Figure 1). If $n \equiv 1(\bmod 2)$, using Lemma 2.6, after $(n-r) / 2$ steps, we get $C_{r}$, by Lemma 2.6 and 2.5 we have $\eta(U(r, n-r))=\eta\left(C_{r}\right)=0$. If $n \equiv 0(\bmod 2)$, using Lemma 2.6, after $(n-2) / 2$ steps, we get a $P_{2}$, by Lemmas 2.6 we have $\eta(U(n, r))=\eta\left(P_{2}\right)=0$.

Case 2. $k=n-r-1$. Let $U=U(r, 1, k+1)$ (see Figure 5), where $r+k+1=n$, using Lemma 2.5, after $(r+1) / 2$ steps, we get $k K_{1}$, by Lemmas 2.3 we have $\eta(U(r, 1, k+1))=\eta\left(k K_{1}\right)=k=n-r-1$.

Case 3. $1 \leq k \leq n-r-2$. Let $U=U(r, l, k+1)$ (see Figure 3), where $r+l+k=n$. If $n \neq k(\bmod 2)$, Using Lemma 2.6, after $l / 2$ steps, we get $C_{r} \cup k K_{1}$, by Lemmas 2.3 and 2.5 we have $\eta(U)=\eta\left(C_{r} \cup k K_{1}\right)=\eta\left(C_{r}\right)+\eta\left(k K_{1}\right)=k$. Similarly, If $n \equiv k(\bmod 2)$, we have $\eta(U(r, l, k+1))=\eta\left(k K_{1}\right)=k$.

Theorem 3.4 The nullity set of $U_{0,2}(n, r)$ is $\{0,1,2, \cdots, n-r\}$.

Proof. Similar to Theorem 2.3, if $l \equiv 1(\bmod 2)$, we consider the graph $U(r, l, k)$ with $k$ pendants (see Figure 3 ), where $r+l+k-1=n$. If $l \equiv 0(\bmod 2)$, we consider the graph $U^{\prime}(r, l, k)$ with $k$ pendants (see Figure 4), where $r+l+k=n$.

If we take $r=3$ in Theorem 2.3 and $r=4$ in Theorem 2.4, then we have the following Corollary:

Corollary 3.9 [18] The nullity set of $U_{n}$ is $\{0,1,2, \cdots, n-4\}$. 


\section{Acknowledgements}

This work is supported by the Natural Science Foundation of Qinghai Province (Grant No. 2011-Z-911).

\section{References}

[1] Von Collatz, L. and Sinogowitz, U. (1957) Spektren Endlicher Grafen. Abhandlungen aus dem Mathematischen Seminar der Universität Hamburg, 21, 63-77. http://dx.doi.org/10.1007/BF02941924

[2] Longuet-Higgins, H.C. (1950) Resonance Structures and MO in Unsaturated Hydrocarbons. The Journal of Chemical Physics, 18, 265-274. http://dx.doi.org/10.1063/1.1747618

[3] Cvetkovic, D., Doob, M. and Sachs, H. (1980) Spectra of Graphs. Academic Press, New York.

[4] Cvetkovic, D.M. and Gutman, I. (1972) The Algebraic Multiplicity of the Number Zero in the Spectrum of a Bipartite Graph. Matematički Vesnik, 9, 141-150.

[5] Cvetkovic, D.M., Gutman, I. and Trinajstic, N. (1972) Graph Theory and Molecular Orbitals, II. Croatica Chemica Acta, 44, 365-374.

[6] Fiorini, S., Gutman, I. and Sciriha, I. (2005) Trees with Maximum Nullity. Linear Algebra and Its Applications, 397, 245-251. http://dx.doi.org/10.1016/j.laa.2004.10.024

[7] Sciriha, I. (1998) On Singular Line Graphs of Trees. Congressus Numeratium, 135, 73-91.

[8] Sciriha, I. and Gutman, I. (2001) On the Nullity of Line Graphs of Trees. Discrete Mathematics, 232, 35-45.

[9] Hu, S., Tan, X. and Liu, B. (2008) On the Nullity of Bicyclic Graphs. Linear Algebra and Its Applications, 429, 13871391. http://dx.doi.org/10.1016/j.laa.2007.12.007

[10] Li, J., Chang, A. and Shiu, W.C. (2008) On the Nullity of Bicyclic Graphs. Match Communications in Mathematical and in Computer Chemistry, 60, 21-36.

[11] Li, S. (2008) On the Nullity of Graphs with Pendent Vertices. Linear Algebra and Its Applications, 429, $1619-1628$. http://dx.doi.org/10.1016/j.laa.2008.04.037

[12] Li, W. and Chang, A. (2006) On the Trees with Maximum Nullity. Match Communications in Mathematical and in Computer Chemistry, 56, 501-508.

[13] Nath, M. and Sarma, B.K. (2007) On the Null-Spaces of Acyclic and Unicyclic Singular Graphs. Linear Algebra and Its Applications, 427, 42-54. http://dx.doi.org/10.1016/j.laa.2007.06.017

[14] Tan, X.Z. and Liu, B.L. (2005) On the Nullity of Unicyclic Graphs. Linear Algebra and Its Applications, 408, $212-220$. http://dx.doi.org/10.1016/j.laa.2005.06.012

[15] Sciriha, I. (1998) On the Contruction of Graphs of Nullity One. Discrete Mathematics, 181, 193-211. http://dx.doi.org/10.1016/S0012-365X(97)00036-8

[16] Sciriha, I. (1999) On the Rank of Graphs. In: Alavi, Y., Lick, D.R. and Schwenk, A., Eds., Combinatorics, Graph Theory and Algrithms, (2), New Issue Press, Western Michigan University, Kalamazoo, 769-778.

[17] Cheng, B. and Liu, B. (2007) On the Nullity of Graphs. Electronic Journal of Linear Algebra, 16, 60-67.

[18] Ashraf, F. and Bamdad, H. (2008) A Note on Graphs with Zero Nullity. Match Communications in Mathematical and in Computer Chemistry, 60, 15-19. 